\title{
Smart Protection System for Medium Voltage Using Wireless Network
}

\author{
Zaid Abdulzahra Jabbar \\ Assistant Lecturer, Ministry of Education, Iraq ${ }^{1}$
}

\begin{abstract}
The development of energy systems to the paradigm of the intelligent protection system strongly depends on the wireless response for the feedback system. To achieve this goal, new infrastructures, technologies, and applications need to bearer increasingly needed. This paper introduces an intelligent switching infrastructure that includes a large, range of possible services for the automation and management of distribution networks. The proposed architecture is based on a wireless monitoring and feedback control platform that enables communication with the intelligent platform. On the one hand, and provides the necessary interfaces for the services of the distribution network in the other. While a large number of applications can be created typical of intention, in this document the focus will be in real-time, the role will present the key function of the monitoring solution for scalability, interoperability, and flexibility, and to enable the integration of different services for the automation of the distribution system. Focus on how to design a smart protection layer based on a wireless system with a smart control platform an example of the coordinated operation of various services of the distribution network via the platform.
\end{abstract}

Keywords: Smart Protection, platform, Android, Mechanism switch

\section{INTRODUCTION}

The smart grid will open up new opportunities for relay protection development, and new sensor technology is being used in smart grids. grid. Simplified data protection algorithm that reduces data processing time. With the launch of the State Grid Corporation of construction of smart grids, smart grids caused by the network reconfiguration characteristics, access to distributed electricity technologies such as work in micro grids to increase new requirements for relay protection based on local measurements information and a small amount of regional information make it difficult to solve these problems using conventional means of protection. Problems; at the same time, the research and application of new technologies (such as new sensor technology, clock synchronization, and data synchronization technologies, information technologies, fiber optic communication technologies, etc.) have provided a wide range of space for the development of relay protection. Adaptive protection means that protection must adapt to changing system conditions, On the software side, the use of a relatively new frequency measurement method, dynamic monitoring of frequency changes, real-time sampling rate adjustment for sampling; and using a new algorithm that improves data accuracy and simplifies hardware circuits. The adaptive principle applied to microprocessor-based line protection, adaptive instantaneous overcurrent protection, over-current protection principles, etc., to meet the requirements of the rapid change operation mode to improve the execution of line protection. Relay protection must adapt to frequent changes in the mode of operation of the power supply system, correctly eliminate various faults and equipment, and the adaptive protection of the relay supports a system of standard functions in the case modification of parameters. The simulation results show that this is an effective adaptive method [1].

The relay protection of a computer must have a hierarchical configuration of the communication lines for the exchange of information with a computer network of other devices. At present, fibre optic communication lines are the most suitable for the transfer of large amounts of information. Transfer and conversion to adaptive protection. Adaptive defence is a defence theory, according to this theory, which allows you to customize the various protection functions, making them more suitable for the practical operation of the power supply system[2].

The key idea is to make some changes to the protection system in order to respond to load changes such as power outages caused by switching or modifying the power system. Adaptive protection as defined in the relevant literature: "Adaptive protection is the basic principle of protection, thanks to which the relay can be automatically adapted to different types of protection. functions or modifications to more suited to the conditions of the power system. ". In addition to normal protection, but it must also have clear adaptive functional modules, and only in this case. it can be called adaptive protection. for the general adaptive protection capability and detection of some aspects of complex failures, there are some limitations, microprocessor hardware circuit [3]. 


\section{IJARCCE}

\section{International Journal of Advanced Research in Computer and Communication Engineering}

Vol. 10, Issue 7, July 2021

DOI 10.17148/IJARCCE.2021.10702

\section{RELATED WORKS}

Utilities are interested in its optimal performance power lines for a better return on investment, electricity markets are becoming more competitive. IN the operation of transmission lines is governed by their assessments. This section describes transmission line estimates. philosophy and overload protection systems. The measured voltage is converted to DC voltage and set the value through the comparator level, the output of which is transmitted to the relay using intelligent control to activate the trip mechanism. Unlike other switches like Circuit breakers, electronic switches have fast activation. machine overload, lower maintenance costs, are manufactured using a small amount of cheap electronic components It is preferable to be sensitive to sensitive loads overcome by installing instant switches [4].

\section{A. Protection automation capabilities}

The smart grid system is equipped with a key network simulation model with two simulated voltage levels to be used for the development and testing of the baseline and advanced protection concepts. using the current-voltage parameter to sense the error then give the smart signal for the system to get action wireless and smartly[3]

\section{B. Remote Terminal Unit for the decentralized generating Simulation}

Along with pure automatic protection, integration Freely adjustable remote control, The system is the main aspect of the smart grid lab. This allows not only to take into account clearly defined values load on the grid but also the situation with energy sources such as wind energy, photovoltaic power, CHP, RTU simulation.

\section{III.NETWORK -AUTOMATION: - OPERATING PRINCIPLE}

The Smart Grid is a distributed system whose domains cover the most traditional sectors of mass production, transport, distribution, consumers, markets, and electricity

electronically, with increasing penetration of relatively new sectors such as renewable energy sources, electric vehicles, and goods compatible with the request and response. Smart Grid Control lets you write recipes for the relationships and interactions between these traditional and new areas in the right manner [5].

directly, in the right place, and in the right way, The purpose of network automation is to enable the creation of utilities flexible control of distribution systems that can be used to improve the efficiency, reliability, and quality of electrical service. Network automation must be based on smart substations as well as in well-developed information and communication technology (ICT) to support automated solutions. In terms of protection of medium voltage supply, the system for automatic isolation of the disconnected part of the power supply and vice versa feed the rest as soon as possible so that

minimize[6].

\section{A. Conventional Protective Devices}

In addition to fuses, which are mainly used to protect the radii

distribution systems, protective relays together with circuit breakers used to protect transmission and sub-transmission systems from various types of damage. These relays are equipped with electricity and voltage transformers (CVT) to measure actual current and voltage systems. Regarding the type of relay and its application, there are many error detection algorithms and protection schemes that can be developed in terms of protection, such as protection from currents, distance

protection, etc. When an error is detected and classified, the relay sends the trip signal on the switch to isolate the area damage from the rest systems.[7].It is called the ability of the switches to break the short circuit current switching capacity (SC) of the switch. There are usually several typical BCs for different voltage levels according to The different types of circuit breakers in power systems.

(Circuit Breaker) is used in simplified substations and

provides the functionality of both a switch and a disconnect or within a unit. DCB is more affordable at a lower price, and that

reduced space, WCB - detachable switch (meets all requirements for the switch as well as for disconnection) and HPL, for extremely high efficiency and high current load. HPL circuit Switches provide a higher breaking capacity and are very expensive.

\section{B. High-Level Short Circuit Current Limitation}

Development of power systems and interconnection of electrical networks while increasing the capacity to create services The need for electricity with high reliability has greatly affected the protection system by increasing the short circuit level to different voltages flat. To deal with very high short circuit currents exceeding SC switches in the system, new switches with higher . 


\section{C.Proposed High Current Protection Scheme}

Proposed High Current Protection Program audits activation of remote protection devices for all relays in the substation. A block diagram of the proposed SPP, which is applied HCPA in a substation where very high short circuit currents are very likely. HCPA is written in MU and remote protection devices for all other relays of the substation. TO to improve the proposed algorithm, for example, HCPA is on bus 1 and a short circuit (SHC) occurred in the turn of the system (Lk) very close to the relay (Rk). If the n-th relay detects a malfunction in zone 1 of the distance protection and the SHC is higher than the BC turn switch (Ikm), HCPS is activated immediately the exclusion signal is sent to the $\mathrm{CBC}$ and at the same time, the travel signal sent to Rk 'at the other end of the line to stop signaling an error another substation. The HCPS estimates the magnitude of the short circuit current. and compares with Ik m CBk to calculate the excess current (Is) the distribution protection system. In smart grids, both industrial and home customers are equipped with smart meters. CM can be found in low and medium-voltage distribution networks [fourteen]. SM can measure both voltages and currents. In this article, possible SM applications in HIF, overcurrent, reverse power, arc, and hypotension protection distribution systems are defined [8]. It's time to make every effort to describe, elaborate, and introduce new guidelines, measurements, and solutions algorithms as well as structures and functions power system protection. This is dictated by the dynamics development and distributed production and creation of the Smart power grid. Otherwise, in the near future, there may be great problems ensuring the proper functioning of the power system protection in electrical networks, which are complex in their functionality and configuration. In addition, it comes from drastic changes to the power system protection network Existence[9].

\section{D.The thermal Magnetic circuit breaker}

The circuit breaker consists of a switching mechanism, an arc. fire extinguishers and roadblocks in a compact molded body.

\section{1- Mechanism switch}

Switching mechanism - quick release / quick release, use switching mechanism without termination. Arc extinguisher A series of grid plates are placed parallel between the supports. insulating substance. The slits in the steel plates come out straight over the contacts and draw an arc from the contact moving upwards split camera. Thus, the arc is limited, splits, and fades.

\section{2-Termination block}

The safety switch activation unit is available in two types:

thermomagnetic formula and full magnetic formula. Block termination is provided for each pole of the circuit breaker, and if one pole is activated, all the poles open the circuit at the same time.

\section{3- Positive indication}

The position of the handle allows you to accurately determine if the switch is closed. ON (up), OFF (down), or on (halfway)[10].

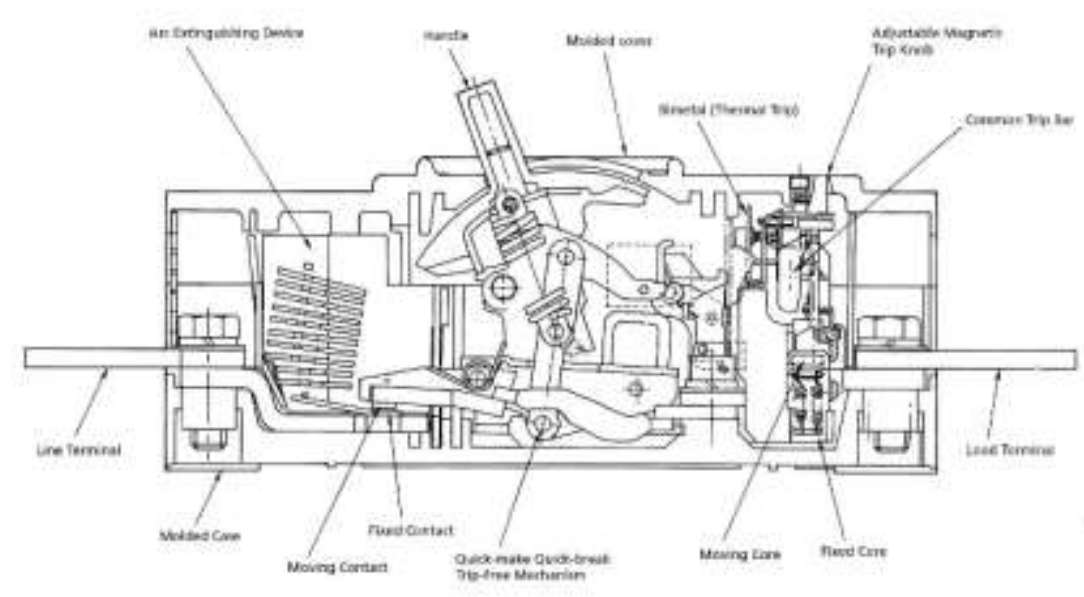

Figure .1.Thermal Magnetic circuit breaker 


\title{
International Journal of Advanced Research in Computer and Communication Engineering
}

\author{
Vol. 10, Issue 7, July 2021
}

DOI 10.17148/IJARCCE.2021.10702

\section{E- Conversion of privately owned protection}

set data in SCL files This section presents a process that is automatically translated own settings in IEC 61850 standard data and saves them as an SCL file. Implementation of the process is facilitated by the proposed code generation process by the authors. The general translation process. The process consists of three main stages: data collection, translation, and export of SCL. The process has is implemented using the Java programming language [11].

Review of SC. The fast performance of a power system or smart grid means its ability to withstand lightning and lightning interference, which ensures the stability and reliability of the existing power supply system. The lightning performance of a power system is based on the performance of each power system but does not simply depend on the performance of each power system[12].

\section{G-Measuring And Monitoring Capabilities}

This study presented and studied a range of electrical systems. protection techniques applied with have, however, focused on devoted to adaptive protection. The techniques were organized using tables. in this review and a comprehensive overview of the techniques This will help researchers conduct literature reviews more efficiently. and find relevant research articles. as good as The categorization of research on adaptive protection is envisaged. the tables give a very short description of the defence approach the methods described in the relevant research papers, and test/simulation methods used. Developments in the field of adaptive protection of electrical systems are underway.

done and research is currently underway for proper optimization

it is a kind of protection[13].

The new generation smart metering circuit usually contains a microcontroller. (MCU.) with analogy front-end interface (.AFE) via analogy to digital converters (A,D,C). This is an architectural structure MS may have measurement and control capabilities.

Displays a list of measurements and monitoring capabilities that usually any SM has or that can be activated. this is It is important to note that, unlike older conventional meters, modern SMs

can measure not only the active power but also various important parameters such as reactive power, apparent power, voltage, and current RMS values (,RMS per second and a half cycle), power factor and harmonics deformation, etc. In addition, with smart function, such as energy-saving settings automatically according to the program, the current CM Supports multiple demand calculation methods, including exclusion, bearing exclusion, synchronization and prediction required[14]

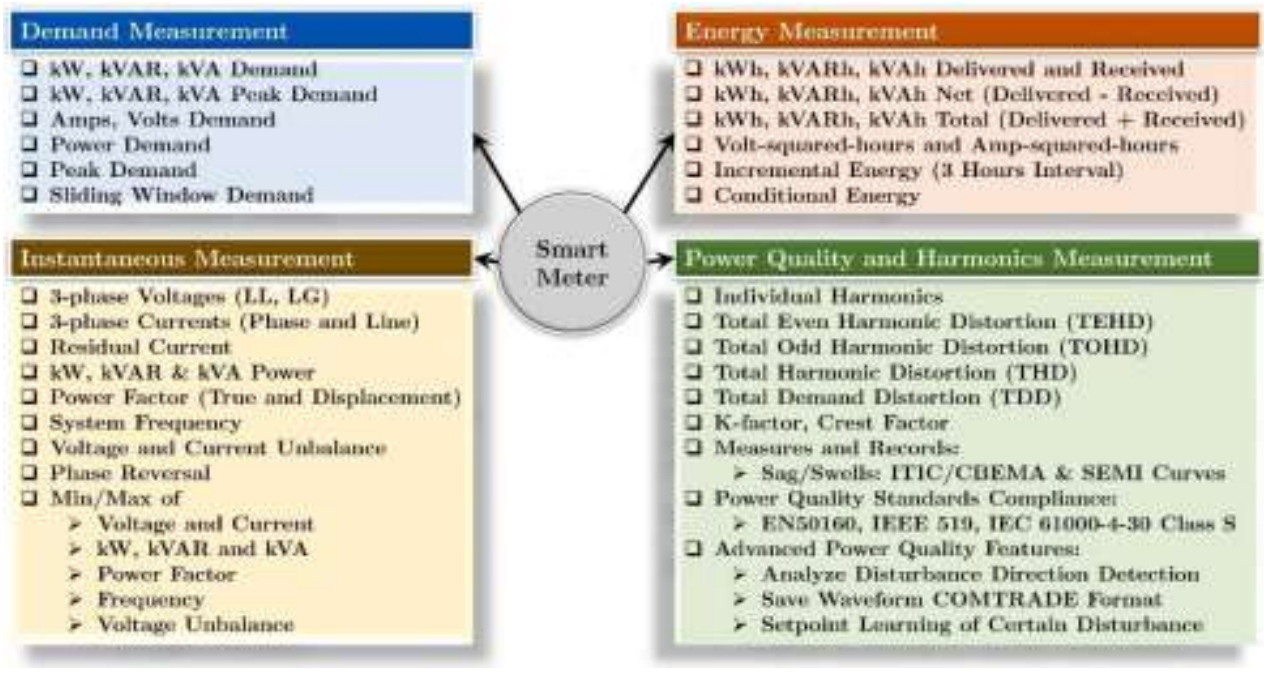

Fig. 2. Measurement and monitoring capabilities of smart meter

The control architecture is shown in Fig. 2 at what level 0 is monitored object, smart meters, FID, and loads (via SST). While the three upper levels are direct control, substation control units, and supervisory network management. Level 1 is real-time.

device controller, level 2 substation energy management system (EMS), and FMS, and level 3 is the main SCADA. Flat are interconnected via a wide area network (WAN) and local area network (LAN), which are configured by OPNET in our booth. Usually, Real-time device controllers are built into the devices. and include a network interface card (NIC) such as FID and SST. It is worth noting that SST and SCB work[15]. 


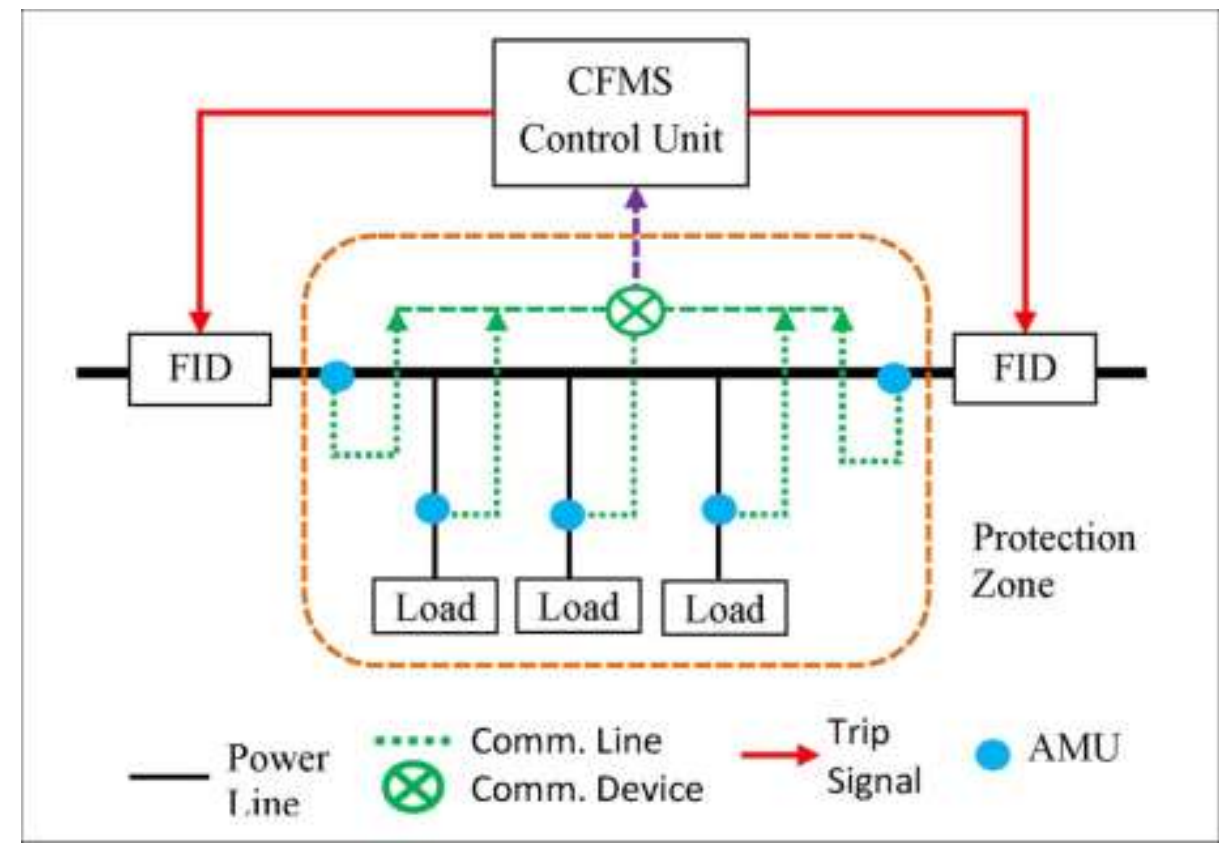

Fig. 3. Within a protection zone, the FIDs and loads are connected to power lines while the CFMS, AMUs, loads (via SSTs) are connected to the communication network. [14]

In the field of Wi-Fi-enabled IoT devices, Kolias et al. showed how IoT devices are sensitive to Man-In-The-Middle (MITM) attacks. In this type of attack, an attacker creates a software access point (SoftAP), also known as a rogue access point, to entice these IoT devices to connect to this rogue access point to monitor the data being transmitted. The authors further describe how the less feature-rich operating systems (O.S.) of many IoT devices find it difficult to distinguish between a legitimate access point and a rogue access point, making these devices vulnerable to this type of attack[16]

\section{IV.DESIGN METHODOLOGY}

This section explains the Hardware and Software designing of protection relay in detail. relay measures single-phase quantities in the power system. level. The current and voltage transformers go down system currents and voltages up to the corresponding value for further processing. Transformers provide electrical and static isolation between the analogy input and internal variables electronic circuits. For two CT configurations: $1 \mathrm{~A} / 2 \mathrm{~mA}$ and 5A / 10mA, enter a current range for desired the protection range is from $90 \%$ to $100 \%$ of the nominal value, From $50 \mathrm{~mA}$ to $200 \mathrm{~A}$. Similar for VT $63.5 \mathrm{~V} / 59 \mathrm{mV}$ is from $90 \%$ to $100 \%$ of the nominal value, from $220 \mathrm{~V}$ to $230 \mathrm{~V}$., This pin is applied to a signal or analogy input air conditioning system.

\section{A. System design}

Given the ubiquitous Wi-Fi traffic between IoT devices, it can be predicted that TX-RX networks will become ubiquitous in the future, which will not allow interference in human activities without devices. homology. Indoors, WiFi signals are transmitted over many routes from TX to RX due to reflection, scattering, and refraction from walls, doors, furniture, and the presence and movement of people. Most Wi-Fi devices are currently equipped with multiple antennas for multiple inputs, multiple outputs and on the physical plane receive rectangles Multiplex division frequency that supports the IEEE $802.11 \mathrm{n} /$ ac standard. IN to increase the data transfer rate, TX splits the data and sends it in a series narrowly subdued at different frequencies simultaneously. Unlike RSS, which records only the overlap of multipath signals, discloses detailed information on how the signal propagates and intervenes, including various time delays, amplitude-phase attenuation, and phase shift of multiple paths in each subsector. Analysis of these signals Dissemination variants caused by human movements makes the recognition of human activity without the use of devices feasible. In short, a signal can be modelled as a channel push response and the receiver can provide a sample version of the signal spectrum of each secondary carrier in the frequency domain containing both amplitude attenuation and phase shift in the form of complex numbers. These measurements. 
Vol. 10, Issue 7, July 2021

DOI 10.17148/IJARCCE.2021.10702

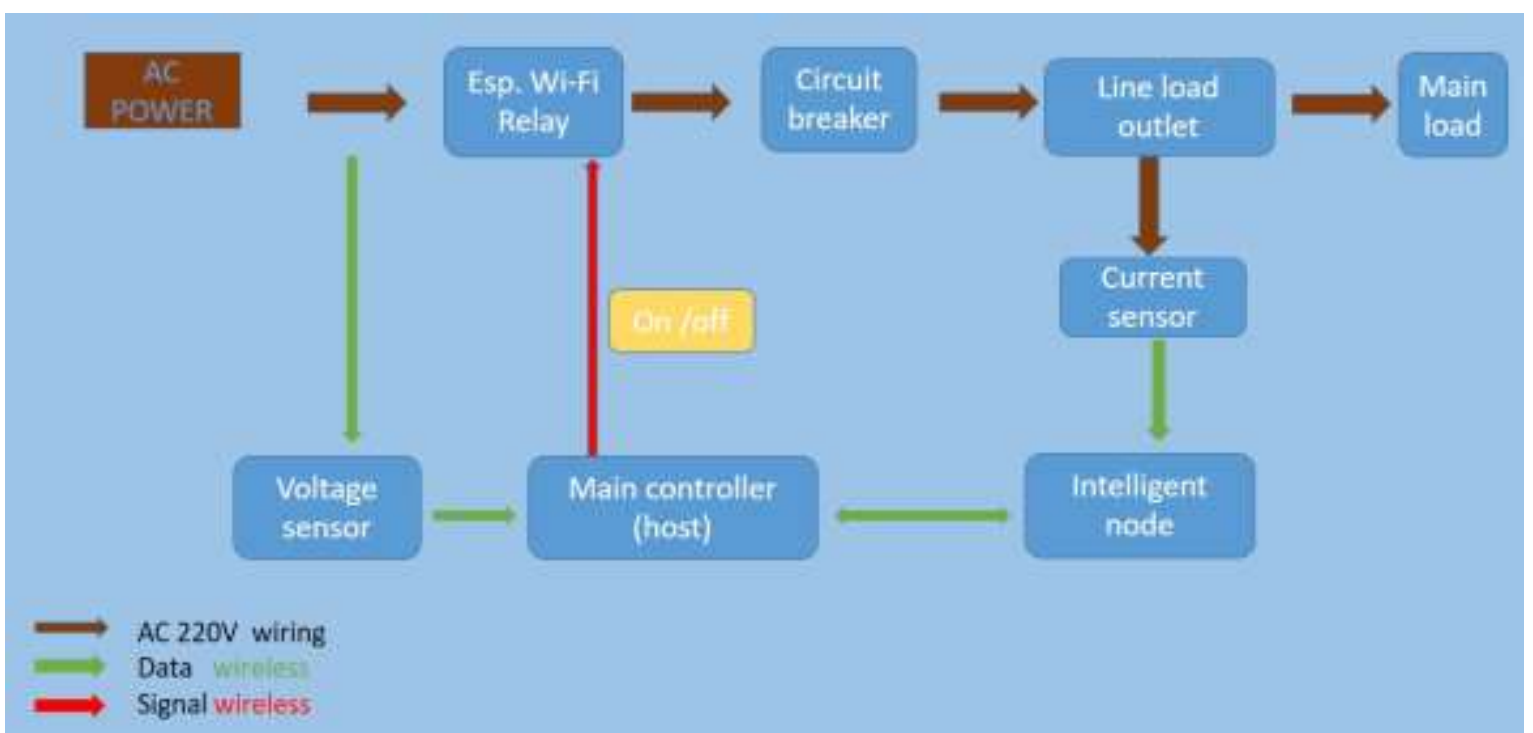

Fig.4.Architecture of smart building-functional block /diagram

\section{B.Hardware design}

We have developed a low-cost three-tier communication architecture to control the MV wireless security system in open fields, as shown in Figure 5. For open fields over a large area, we use a private network between the gateway and the actuator. nodes. to reduce communication costs for farmers and Wi-Fi technologies to connect gateway nodes to servers or mobile networks where Wi-Fi is off One gateway can control multiple actuator assemblies and one actuator assembly can connect multiple designated. A block diagram of the actuator assembly and Fig.4 shows a prototype of the node.

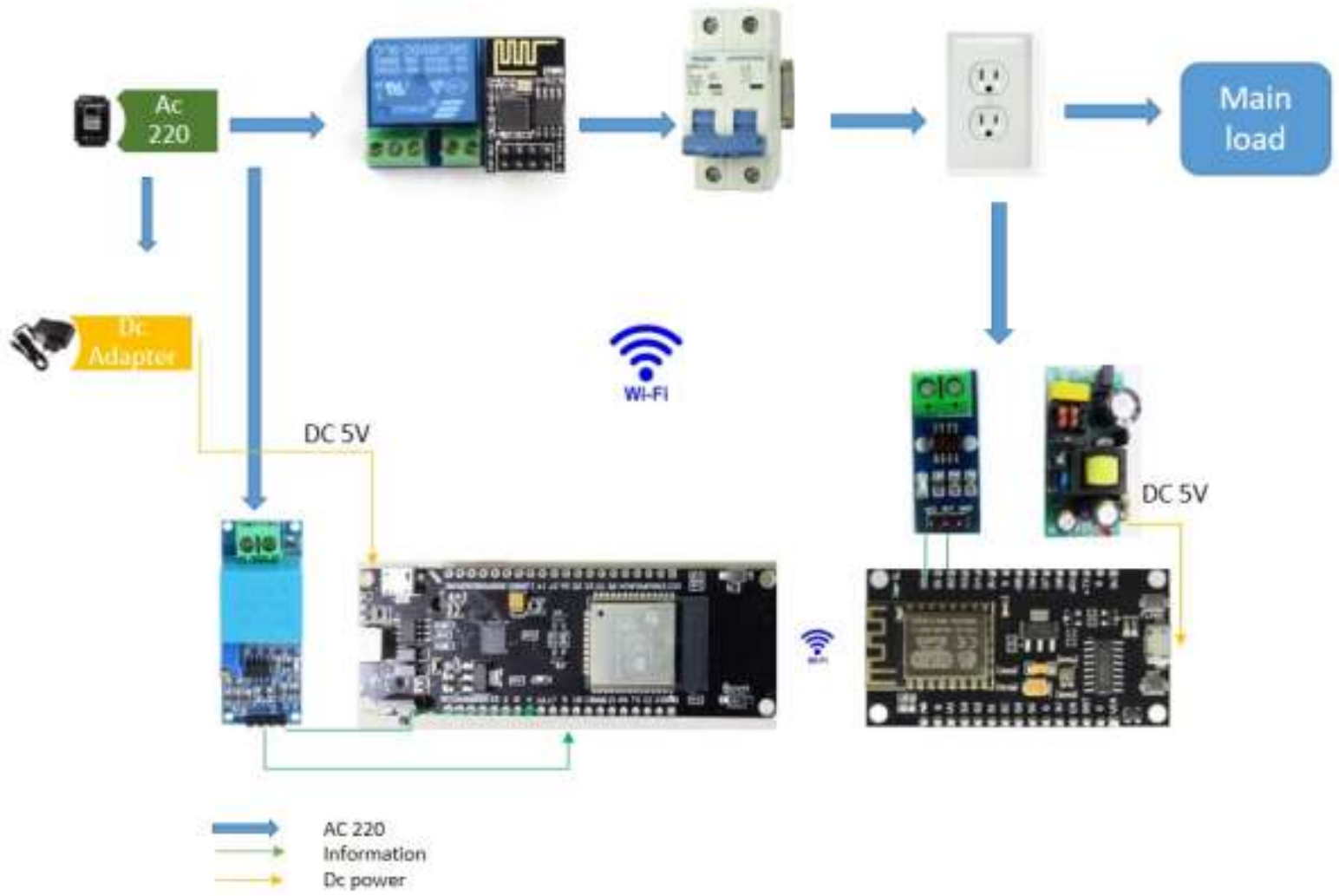

Fig.5 . hardware design system over all 
Vol. 10, Issue 7, July 2021

DOI 10.17148/IJARCCE.2021.10702

\section{C.Esp8266-wifi-module-nodemcu-v3}

The best way to quickly deploy an IoT application with fewer built-in shapes to add - select this "NodeMCU" shape. Today we will give a detailed introduction to NodeMCU V3. It is open-source firmware and development kit that plays a vital role in developing the right IoT product using

multiple script lines. The unit is mainly based on the ESP8266, which is a cheap Wi-Fi microchip. including full TCP / IP stack capabilities and microcontroller. this is provided by the manufacturer Espresso Systems. ESP8266 Node is a sophisticated device that combines some of the features of a regular Arduino board with the ability to connect to the Internet.[17] Arduino units and microcontrollers have always been a great choice include automation in the project. But these sections are coming to a small drawback since they do not have built-in Wi-Fi capability, then we need to add external wifi protocol to these devices to make them compatible with the internet channel.

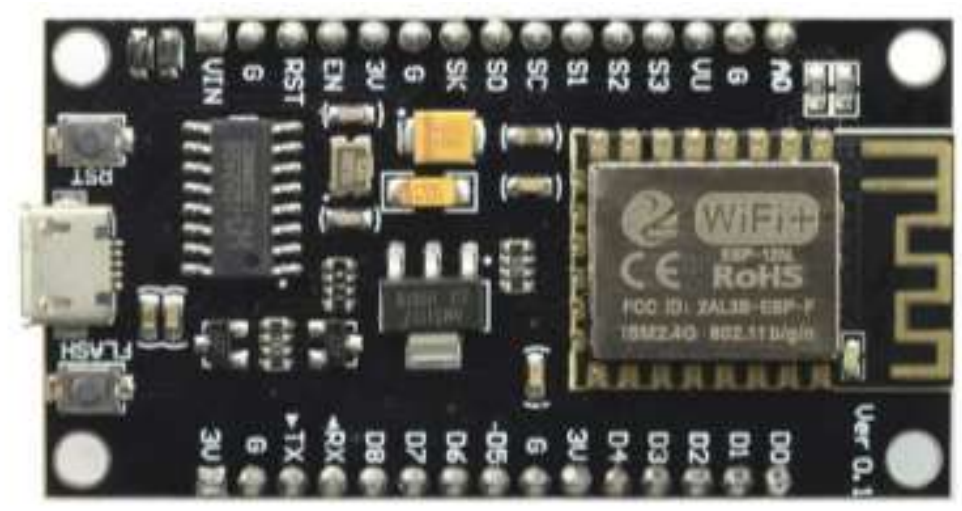

Fig.6. Esp8266-wifi-module

\section{D.ESP-WROOM-32s}

The core of this unit is an expandable and customizable ESP32 chip. Two CPU cores can be controlled separately. The clock frequency is adjustable from $80 \mathrm{MHz}$ to $240 \mathrm{MHz}$ and supports RTOS. It's universal Wi-Fi + BT + BLE MCU module. ESP-WROOM-32s The unit combines 'traditional', low-power lands and Wi-Fi. Wide range of uses: Wi-Fi supports a wide range of communication connections, As well as direct internet connection via a router · Bluetooth, which allows users .Connect to a mobile phone or transfer a BLE Beacon signal to locate a signal. IN The unit supports transmission speeds of up to $150 \mathrm{Mbps}$ and an antenna output of $20 \mathrm{~dB}$ for maximum wireless connectivity. As a result, there is in this section Industry-leading performance and a high degree of integration, Wireless transmission distance, power consumption, and network connectivity[18]

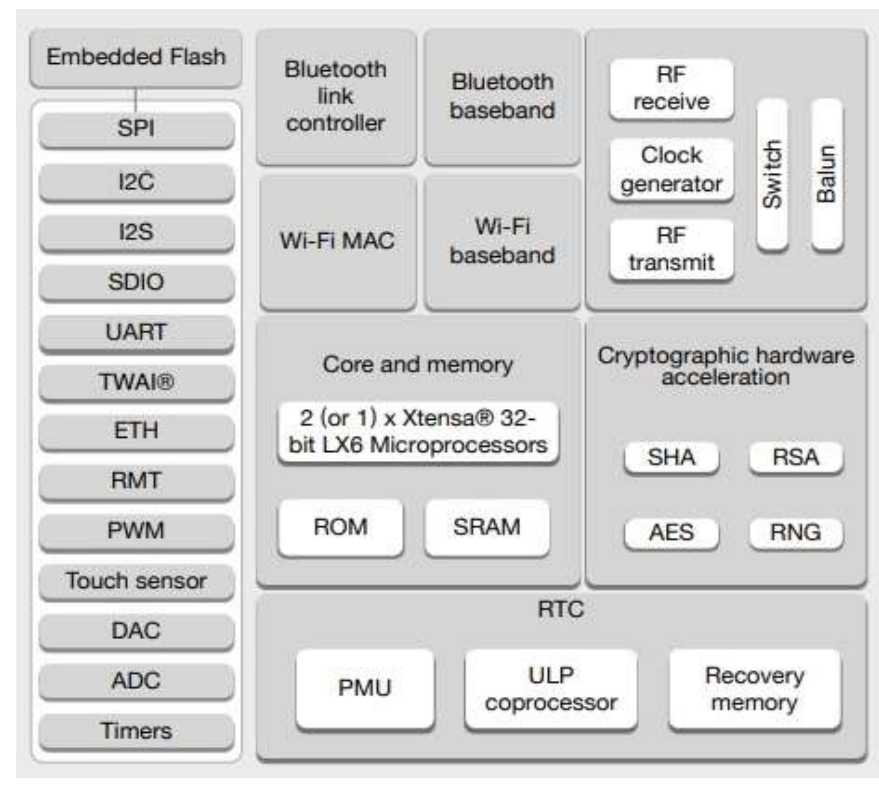

Fig.7. ESP-WROOM-32s Structure 


\section{E.Flow diagram of the gateway node.}

The system constructed for wireless layer protection to avoid short circuit and circuit fail by overload, the system using a Wi-Fi relay board for wireless protection depending on the smart response for the main controller, esp node sensitive to the real-time current in the circuit when current or voltages be up to normal for adjusting in the system esp node sends a signal for the main controller to make switching for main feeder power off by Wi-Fi relay the android app application is the main platform control and monitoring synchronize with the hard ware system by main controller IP address

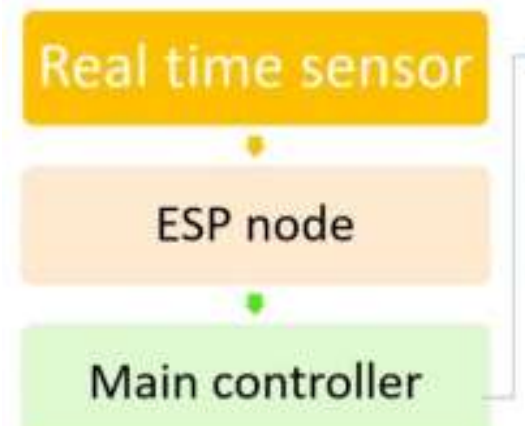

\section{wifi smart relay}

5

switching off line

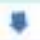

\section{Platform monitoring}

Fig.8. . Flow diagram of the gateway node.

The Intelligent Medium Voltage Protection System is dependent on the Wi-Fi connectivity system of the IEEE 802 family of protocols and is designed to seamlessly connect to a wired Ethernet network. Compatible devices can be networked via wireless access points as well as wired devices and Internet data rates.

the "data values" refer to Wi-Fi. the specifications refer to the primary rate of radio communication, not actual TCP / IP performance. In general, the difference is called the protocol and required by Wi-Fi protocol for conflict management, relaying and general link management. Android application represent as simple and practical platform for monitoring and switching the system functions.

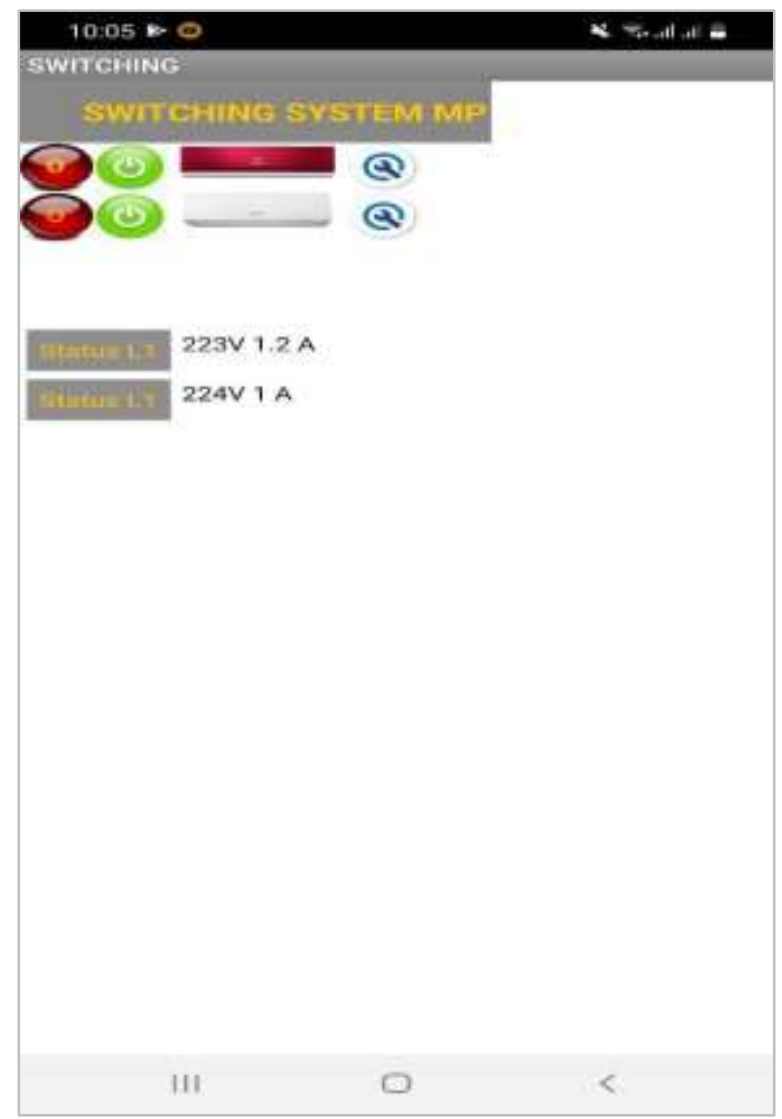

Fig.9.Android platform application 


\title{
IJARCCE
}

\section{International Journal of Advanced Research in Computer and Communication Engineering}

\author{
Vol. 10, Issue 7, July 2021
}

DOI 10.17148/IJARCCE.2021.10702

\section{RESULTS AND DISCUSSION}

On the power line and between the point of failure and the power source power supply there are many protective relays in series. Relays must be well-tuned, with the relay closest to the fault should operate first. The input relays must be configured to operate in series.

provide backup protection even if the relay does not work. It is called selective coordination. To meet these requirements, their ay must be calculated or adjusted to match the minimum current to the minimum year. It must also be selective with other relays in the rest System. If the above criteria are met, adequate equipment protection, personal, manufacturing is complete. Traditional defense coordination cannot be applied here. where there are many current paths in different directions and many taps that cannot recognize the point of failure. Fig. 7 shows the configuration system under study. System parameters: given The relay measures single-phase quantities in the power system. level. The current and voltage transformers go down system currents and voltages up to the corresponding value for further processing. Transformers provide electrical and static isolation between the analog input and internal variables electronic circuits. For two CT configurations: $1 \mathrm{~A} / 2 \mathrm{~mA}$ and 5A / 10mA, enter the current range for desired the protection range is from $90 \%$ to $100 \%$ of the nominal value, From $50 \mathrm{~mA}$ to $200 \mathrm{~A}$. Similar for VT $63.5 \mathrm{~V} / 59 \mathrm{mV}$ is from $90 \%$ to $100 \%$ of the nominal value, i.e. from $220 \mathrm{~V}$ to $230 \mathrm{~V}$. This pin applied to a signal or analog input air conditioning system. Circuit breaker represent in the system as first protection layer then Wi-Fi relay working as smart protection layer.

\section{VI.CONCLUSION}

The selectivity of the relay must be improved as well as the shutdown loads connected to repaired feeders should be minimized. And you succeed that, relays based on wireless technology must fit well. The document introduces a new defense philosophy for detecting damaged areas properly using IEEE 802.16 wireless protocol. The idea has the peculiarity of considering the studied configuration as a zone instead of several zones, see Fig. 5Now the electrical substations should new wireless technologies to address the growing physical security and the complexity of communication networks. These technologies are must be carefully selected to meet the needs of protection and power applications [four]. Wireless technology can be used to deal with many protection problems and many disadvantages that are still integrated to protect power lines. Such a high system The specification will be very suitable for protecting a smart grid in the case of integration of REE and RES in the distribution and transmission network systems.

\section{REFERENCES}

[1] H. Y. Zhang and S. De Li, "Design of adaptive line protection under smart grid," APAP 2011 - Proc. 2011 Int. Conf. Adv. Power Syst. Autom. Prot., vol. 1, pp. 599-603, 2011, doi: 10.1109/APAP.2011.6180471.

[2] S. Dahal and S. Paudyal, "Development of System Integrity Protection Scheme for Real-time Protection of an Overloading Feeder," 2019 9th Int. Conf. Power Energy Syst. ICPES 2019, pp. 0-4, 2019, doi: 10.1109/ICPES47639.2019.9105467.

[3] I. Bielchev, A. Naumann, A. Visyashchev, R. Krebs, and Z. A. Styczynski, "Modeling and investigation of communication and protection scenarios for Smart Grid," IEEE PES Innov. Smart Grid Technol. Conf. Eur., pp. 1-5, 2012, doi: 10.1109/ISGTEurope.2012.6465739.

[4] P. Abirami and M. L. George, "Electronic Circuit Breaker for overload protection," 2016 Int. Conf. Comput. Power, Energy, Inf. Commun. ICCPEIC 2016, pp. 773-776, 2016, doi: 10.1109/ICCPEIC.2016.7557324.

[5] Massoud Amin, A. M. Annaswamy, C. L. Demarco, and T. Samad, "IEEE Vision for Smart Grid Controls: 2030 and Beyond Reference Model," pp. 1-10, 2013.

[6] A. Bosisio, A. Berizzi, A. Morotti, A. Pegoiani, B. Greco, and G. Iannarelli, "IEC 61850-based smart automation system logic to improve reliability indices in distribution networks," APAP 2019 - 8th IEEE Int. Conf. Adv. Power Syst. Autom. Prot., pp. 1219-1222, 2019, doi: 10.1109/APAP47170.2019.9224717.

[7] M. Mahmoudian Esfahani and O. Mohammed, "An intelligent protection scheme to deal with extreme fault currents in smart power systems," Int. J. Electr. Power Energy Syst., vol. 115, no. March 2019, p. 105434, 2020, doi: 10.1016/j.ijepes.2019.105434.

[8] A. Radhakrishnan and S. Das, "Protection Functionalities in Smart Meters to Enhance Distribution System Protection," Int. Conf. Innov. Smart Grid Technol. ISGT Asia 2018, pp. 1038-1043, 2018, doi: 10.1109/ISGT-Asia.2018.8467933.

[9] A. Halinka, P. Rzepka, and M. Szablicki, "Agent model of multi-agent system for area power system protection," Proc. - Int. Conf. Mod. Electr. Power Syst. MEPS 2015, pp. 1-4, 2015, doi: 10.1109/MEPS.2015.7477185.

[10] K. Neribei-cho et al., : "Headquarters," vol. 123, no. 3.

[11] Q. Hong, A. Dyśko, C. D. Booth, V. M. Catterson, S. M. Blair, and T. Rahman, "Translating proprietary protection setting data into standardised IEC 61850 format for protection setting validation," IET Conf. Publ., vol. 2014, no. 626 CP, pp. 1-6, 2014, doi: 10.1049/cp.2014.0068.

[12] C. Tong, Q. Wang, Y. Gao, M. Tong, and J. Luo, "Dynamic Lightning Protection of Smart Grid distribution system," Electr. Power Syst. Res., vol. 113, no. 183, pp. 228-236, 2014, doi: 10.1016/j.epsr.2014.03.012.

[13] H. Khalid and A. Shobole, "Existing Developments in Adaptive Smart Grid Protection: A Review," Electr. Power Syst. Res., vol. 191, no. November 2020, p. 106901, 2021, doi: 10.1016/j.epsr.2020.106901.

[14] S. Chakraborty, S. Das, T. Sidhu, and A. K. Siva, "Smart meters for enhancing protection and monitoring functions in emerging distribution systems,” Int. J. Electr. Power Energy Syst., vol. 127, no. November 2020, p. 106626, 2021, doi: 10.1016/j.ijepes.2020.106626. 
Vol. 10, Issue 7, July 2021

DOI 10.17148/IJARCCE.2021.10702

[15] X. Liu, Z. Cai, H. Fan, and M. Yu, "Experimental studies on the rtEthernet-based centralized fault management system for smart grids," Electr Power Syst. Res., vol. 181, no. May 2019, p. 106163, 2020, doi: 10.1016/j.epsr.2019.106163.

[16] J. O. Agyemang, J. J. Kponyo, G. S. Klogo, and J. O. Boateng, "Lightweight rogue access point detection algorithm for WiFi-enabled Internet of Things(IoT) devices," Internet of Things, vol. 11, p. 100200, 2020, doi: 10.1016/j.iot.2020.100200

[17] A. Al Dahoud and M. Fezari, "NodeMCU V3 For Fast IoT Application Development Lung Sounds analysis View project," ResearchGate, no. October, 2018, [Online]. Available: https://www.researchgate.net/publication/328265730.

[18] V. Version, "Nodemcu - 32s Datasheet," 2019.

\section{BIOGRAPHY}

Zaid Abdulzahra Jabbar is Assistant Lecturer, Ministry of Education, Iraq,M.E. Electronics degree from SCOE college, Savitribai Phule Pune University, received B.S. degree from department of electrical engineering , university of technology - Iraq, interest is in the research areas of Wireless Sensor Networks, Data Communication, Home networks, Green Energy, Smart Home, IEEE 802.11/802.15.4 MAC protocols, ZigBee networks and implementation of real sensor platforms. 